\title{
VLBI MORPHOLOGY OF GHZ-PEAKED SPECTRUM RADIO GALAXIES
}

\author{
D. DALLACASA; C. FANTI** and R. FANTI** \\ Istituto di Radioastronomia-CNR, Bologna, Italy
}

\section{Discussion}

GHz-Peaked Spectrum (GPS) radio sources are intrinsically small $(<1 \mathrm{kpc})$ and unbeamed objects. The galaxies considered here $(0316+161,0404+768,0428+205$, $1323+321,1358+624,1819+39,1829+29)$ have been selected from the Peacock and Wall (1981) catalogue, and belong to a complete sample of Compact SteepSpectrum (CSS) radio sources (Fanti et al., 1990). Their radio spectra show a turnover which could be explained in terms of synchrotron self-absorption. It occurs at frequencies ranging from about $100 \mathrm{MHz}$ to $5 \mathrm{GHz}$ and for this reason they do not appear in the 3CR catalogue.

The 3CR CSS galaxies generally show Double morphology (lobes) on the 1 to 10 kpc scale (Fanti et al., 1990). Faint cores are found at high frequencies (e.g. 15 $\mathrm{GHz}$ ) for most of them (van Breugel et al., 1984 and 1992), but in all cases they account for only a very tiny fraction of the total flux density $(<0.5 \%)$. Jets are rare, so that the radio emission is generally lobe-dominated.

GPS galaxies instead show in most cases cores (or candidates), and bright jets even at $1.66 \mathrm{GHz}$ (Dallacasa et al., submitted), while the fractional flux density in the lobes is generally less relevant than in 3CR CSS's. The low-frequency selected 3CR CSS's are probably biassed towards lobe dominated morphologies, with overall sizes and component sizes larger than in GPS radio sources and with the turnover in the spectrum occurring at lower frequencies. The evolutionary scenario in which the GPS galaxies will become CSS's when the source expands and the central engine progressively turns off, is not adequate, since extended radio emission has been found in 20\% of GPS's, and the age of GPS's turns to be of the same order of the extended radio sources when modelling the source expansion in a clumpy medium (see also Carvalho, these proceedings).

\section{References}

van Breugel W.J.M., Miley G.K., Heckman T.M.: 1984, Astron. J., 89, 5.

van Breugel W.J.M., Fanti C. and R., Stanghellini C., Schilizzi R.T., Spencer R.E.: 1992, Astron. Astrophys., $256,56$.

Fanti R. and C., Schilizzi R.T., Spencer R.E., Nan Rendong, Parma P., van Breugel W.J.M, Venturi T.: 1990, Astron. Astrophys., 231, 333.

Peacock J.A. and Wall J.V.: 1981, M.N.R.A.S., 194, 331.

* also Dipartimento di Astronomia, Bologna. Present address: NFRA, Dwingeloo, NL.

** also Dipartimento di Fisica, Bologna.

T. J.-L. Courvoisier and A. Blecha: Multi-Wavelength Continuum Emission of AGN, 425.

(C) 1994 IAU. Printed in the Netherlands. 